\title{
Efecto del enriquecimiento ambiental sobre la ansiedad: estudio experimental en un modelo animal
}

Effect of environmental enrichment on anxiety: experimental study in an animal model
Author:

Marian Andrea Suarez Rodriguez ${ }^{1}$

Silvana Paola Pulido Barrera ${ }^{1}$

Santiago José Duran Sira ${ }^{1-2}$

Peter Joel Caicedo-Pinto ${ }^{1-2^{*}}$

Dafael Armando Bonfante-Cabarcas ${ }^{1}$

\section{INVESTIGACIÓN CIENTÍFICA}

Cómo citar este artículo:

Suarez M, Pulido S, Duran S, Caicedo-Pinto P, Bonfante-Cabarcas R Efecto del enriquecimiento ambiental sobre la ansiedad: estudio experimental en un modelo animal, Innovaciencia 2021; 9(1), 18-33.

DOI: https://doi.org/ 10.15649/2346075X.2563

Fecha de recepción:

Recibido: 01 de octubre de 2021

Aceptado: 15 de diciembre de 2021

Publicado: 17 de diciembre de 2021

Palabras clave:

Enriquecimiento Ambiental; Ansiedad; Hiperactividad; Pruebas no condicionadas de Ansiedad; Preferencia por Sacarosa.

Keywords:

Environmental Enrichment; Anxiety; Hyperactivity; Unconditioned anxiety test; Preference for Sucrose.

\section{RESUMEN}

La ansiedad es un problema de salud mundial que amerita el estudio de nuevas alternativas para su tratamiento terapéutico, entre ellas el enriquecimiento ambiental (EA). El presente trabajo estudió el efecto del EA sobre la ansiedad. 20 ratones C57BL/ 6 fueron divididos en 2 grupos: Grupo Control (GC, $n=10$ ) y Grupo Experimental (GE, $n=10)$. El GE recibió EA basado en estímulos sensoriales (olfativos, gustativos, visuales y táctiles) y nichos oscuros, mientras que el GC fue hospedado individualmente en un ambiente restringido de EA. Los ratones fueron evaluados en el Laberinto Elevado en Cruz (LEC), Cámara Luz/Oscuridad (CLO) y mediante la prueba Preferencia por Sacarosa (PS). El EA causó hipermotilidad en LEC con preferencia por los desplazamientos entre brazos cerrados $(\mathrm{p}<0.05)$; aumentó el tiempo unitario de permanencia en ambos compartimientos de CLO $(\mathrm{p}<0.05)$ e incrementó significativamente el consumo de sacarosa en PS ( $\mathrm{p}<0.05)$. En conclusión; la técnica del EA basado en incluir nichos oscuros induce hiperactividad con niveles de ansiedad disminuidos, permitiendo la expresión de conductas instintivas.

\section{ABSTRACT}

Anxiety is a global health problem; therefore, it is necessary to study new therapeutics alternatives, including environmental enrichment (EE). In the present paper the effect of EE on anxiety were studied. EE was evaluated in 20 C57BL/6 mice divided into 2 groups: Control Group $(\mathrm{CG}, \mathrm{n}=10)$ and Experimental Group (EG, $n=10)$. EG received EE with various sensory stimuli (olfatoy, gustatory, visual and tactile) and dark niches. CG was housed individually in a restricted environment. Mice were evaluated in the Elevated Plus Maze (EPM), Light/Dark Box (LDB) and Preference for Sucrose (PS). EE caused hypermotility in EPM with preference for shifts between closed arms $(p<0.05)$, increased the unit residence time in both compartments of LDB $(p<005)$ and significantly increased the PS $(p<0.05)$. In conclusion; the EE based on including dark niches induces hyperactivity with decreased levels of anxiety, allowing the expression of instinctive behaviors to dark places.

* Autor de correspondencia: Peter Caicedo-Pinto. Email: pcaicedo88@gmail.com

1 Unidad de Bioquímica, Decanato de Ciencias de la Salud, Universidad Centroccidental "Lisandro Alvarado", Barquisimeto, Venezuela.

2 Departamento de Ciencias de la Conducta, Decanato de Ciencias de la Salud, Universidad Centroccidental "Lisandro Alvarado", Barquisimeto, Venezuela. 


\section{INTRODUCCIÓN}

Los trastornos de ansiedad son condiciones de salud que implican cambios en los pensamientos, emociones y comportamientos de un individuo. Los determinantes de estas alteraciones incluyen no solo atributos individuales, como los mencionados anteriormente, sino también factores sociales, culturales, económicos, políticos y ambientales. El estrés, la genética, la nutrición, las infecciones perinatales y la exposición a riesgos ambientales, son factores que contribuyen a la aparición de dichos trastornos [1].

Según datos recopilados por la Organización Mundial de la Salud, los trastornos de ansiedad se posicionan como el segundo trastorno mental con mayor número de discapacidad en la Región de las Américas con un $2.1 \%$ de años de vida ajustados en función a la discapacidad y $4.9 \%$ de años perdidos por discapacidad. En tal sentido, se estima que la transición o desarrollo epidemiológico de estas alteraciones está transformando el sistema de salud, puesto que, los determinantes de las necesidades mundiales en materia de salud son predominadas por las enfermedades no trasmisibles tales como dichas alteraciones [2].

La ansiedad se describe como un estado psicológico, fisiológico y conductual inducido por una amenaza para el bienestar o la supervivencia de un individuo, ya sea real o potencial. Se caracteriza por un aumento de las conductas expectantes con agitación, acompañado del incremento de las actividades autonómicas y neuroendocrinas que activan patrones de comportamiento específicos e incluyen una transición conductual desde comportamientos continuos, como por ejemplo exploración o alimentación, a conductas defensivas (inmovilidad) o de escape (huida). La función de estos cambios es facilitar el manejo de una situación adversa o inesperada. Sin embargo, si la función adaptativa de la ansiedad no tiene éxito y la respuesta anticipatoria ante una amenaza futura se vuelve excesiva de acuerdo al contenido de los pensamientos o creencias asociadas, puede convertirse en un estado patológico reiterativo, que interfiere con la vida cotidiana y con la capacidad de hacer frente a los desafíos de eventos estresantes de los sujetos [3,4].

Los trastornos de ansiedad se pueden tratar con terapias farmacológicas (ansiolíticos y antidepresivos) y psicológicas (terapia cognitivo-conductual). En tal sentido, la combinación de estos elementos son un punto clave para controlar la sintomatología de estos trastornos, sin embargo, el costo y los efectos colaterales adictivos del tratamiento farmacológico representan un obstáculo al momento de tomar las decisiones terapéuticas [5,6], por lo tanto, las terapias alternativas que asocien las terapias cognitivo-conductuales con el enriquecimiento ambiental (EA) podrían ser interesantes.

El término EA se refiere a una mejora de las condiciones de confinamiento de animales (de laboratorio o no) en comparación con los alojados de forma estándar. Estas condiciones incluyen sitios de alojamiento o jaulas más grandes que contienen objetos y diferentes espacios que facilitan el ejercicio, el juego y la exploración, al tiempo que permiten a los animales un mayor control sobre su entorno. En algunos paradigmas experimentales, el EA también se puede realizar aumentando el número de animales por jaula o sitio de alojamiento, favoreciendo así las interacciones sociales. Algunos estudios recientes han optado por el uso de espejos con el objetivo de simular la estimulación social, observando un efecto positivo en los niveles de exploración y ansiedad [7]; en tal sentido, todos estos elementos promueven la estimulación cognitiva, sensorial, motora y social de los sujetos, aumentando su respuesta adaptativa a entornos desconocidos [8,9].

El EA ha sido identificado como un factor protector en el tratamiento de algunos trastornos emocionales relacionados con el estrés, como por ejemplo la depresión. No obstante, los efectos ansiolíticos inducidos por

\section{Innovaciencia}


el EA no son tan claros; en general, la exposición al EA produce efectos similares a los fármacos ansiolíticos en diferentes modelos animales destinados a investigar trastornos relacionados con la ansiedad, sin embargo, otros estudios mostraron efectos parciales o resultados negativos. Se ha planteado una hipótesis que afirma que el EA actúa como un agente estresante leve por los desafíos cognitivos que incluye. Asimismo, la introducción repetida de nuevos objetos y la oportunidad de explorar libremente ambientes cambiantes es comparable a las exposiciones repetidas a un estímulo estresor. De hecho, algunos estudios han demostrado efectos de tipo ansiogénico en animales expuestos a ambientes enriquecidos, quienes presentaban elevados niveles de corticosterona, neofobia a la sacarosa o una mayor actividad autonómica [10]. No obstante, el EA se ha propuesto como una posible estrategia para aumentar progresivamente la capacidad cognitiva en algunos modelos animales y en infantes dentro de un contexto educativo, mejorando la adquisición y consolidación de la memoria, el aprendizaje y ayudando a un mayor y más rápido proceso adaptativo facilitando el fenómeno de habituación $[9,11]$.

Teniendo en cuenta que las psicoterapias actuales para los trastornos relacionados con la ansiedad incluyen un tratamiento farmacológico que en algunos casos resulta contraproducente debido a los efectos secundarios de los fármacos utilizados, y a pesar de los resultados contradictorios mencionados anteriormente del EA como terapia alternativa, resulta importante conocer su efecto sobre la ansiedad, por lo que en el presente trabajo estudiamos el efecto del EA con nichos oscuros y diversos estímulos sensoriales (auditivos, olfativos, gustativos, visuales y táctiles) sobre la ansiedad en ratones C57BL/6, utilizando una batería de pruebas no condicionadas como el laberinto elevado en cruz y la caja de luz-oscuridad, así como el ensayo de preferencia por la sacarosa.

\section{MATERIALES Y MÉTODOS}

Población y muestra. Estuvo constituida por 20 ratones hembras de la cepa C57BL/ 6 con un peso promedio 17,90 \pm 0,33 gr, que fueron obtenidos del Bioterio Central de la Universidad Centroccidental "Lisandro Alvarado", Barquisimeto (Venezuela). Los ratones fueron seleccionados de manera aleatoria y colocados en 2 grupos de 10 ratones c/u denominados grupo Control (GC) y grupo experimental (GE). La totalidad de los ratones del grupo GE fueron colocados en una jaula de acero inoxidable de 50 x 36 x $13 \mathrm{~cm}$; mientras que los ratones del grupo GC fueron acomodados en una jaula dividida en 10 celdas de 14 x 9 x $14 \mathrm{~cm} \mathrm{c} / \mathrm{u}$, a razón de 1 ratón por celda para evitar cualquier tipo de relación ente ellos, entendiéndose que al dejar a todos los animales de dicho grupo dentro de una misma jaula se estaría promoviendo un tipo de EA sensorial y social. Los ratones tuvieron libre acceso al agua destilada y comida, que consistió en alimento comercial peletizado. Se mantuvieron a una temperatura ambiente entre 25 y $30^{\circ} \mathrm{C}$ y ciclos luz/oscuridad de 12 horas c/u.

Enriquecimiento Ambiental (EA). Consistió en colocar armónicamente los siguientes objetos dentro de las jaulas: puentes de cartón que unían rampas de plástico, túneles de cartón o de plástico con paredes horadadas que terminaban en túneles conectores en forma de $\mathrm{T}$ de acero inoxidable, madrigueras de plásticos con dos orificios para el acceso y cáscaras de arroz en el piso, balancines atados al techo de la jaula y canicas de color negro. Se les proporcionó estímulos sensoriales alternados diariamente, tales como: olores (cereza, lavanda, maresía y menta) colocados en motas de algodón introducidas en frascos de plástico de 5 cc con orificio de $1.3 \mathrm{~cm}$ de diámetro; sabores (ácido, amargo, dulce, salado, umami) que fueron mesclados en bocadillos de merengues, cotufas o maíz inflado y colocados en los balancines; visuales que consistían en balancines de diversos colores (rojo, amarillo, verde y azul) y en luces coloridas intermitentes colocadas durante la noche, y estímulos táctiles como piedras pequeñas rugosas, cáscaras de arroz o virutas de madera en el piso. 
El GC fue colocado en un espacio restringido con piso cubierto de cascaras de arroz y libre acceso al agua y comida. Los animales fueron hospedados en los ambientes descritos durante un periodo de 2 meses previo a las pruebas conductuales y durante el desarrollo de las mismas.

Protocolo experimental. Constó de una sola sesión aplicada en una semana en la que realizaron 2 pruebas de ansiedad no condicionadas (LEC y CLO) y la prueba de preferencia por sacarosa (PS). Los ensayos se ejecutaron posteriormente a los 2 meses de alojamiento en un ambiente enriquecido para el GE y en las celdas individuales para el GC. Las pruebas no condicionadas de ansiedad se aplicaron alternando los grupos con un día de por medio y siguiendo la siguiente secuencia: LEC, CLO; por último, la prueba de PS se llevó a cabo después de un periodo de adaptación de los animales y de manera simultánea para ambos grupos.

Laberinto elevado en cruz (LEC). Este ensayo se realizó en un laberinto de cuatro brazos colocado a una altura de $81 \mathrm{~cm}$, compuesto por dos brazos abiertos (sin paredes), con dimensiones de $50 \mathrm{~cm}$ de largo por 9,3 cm de ancho; dos brazos cerrados de iguales dimensiones con paredes de $40 \mathrm{~cm}$ de alto y un área central de $5 \times 5 \mathrm{~cm}$. El material de construcción fue de madera, pintada de negro mate. El observador se encontraba a una distancia de $50 \mathrm{~cm}$ del laberinto. La prueba consistió en colocar al ratón en el brazo abierto con dirección hacia el sur, para que explorara libremente el laberinto, a partir de ese momento se cuantificó el número de desplazamientos de un brazo a otro y el tiempo de permanencia en los mismos durante un periodo de 10 min.

Cámara de luz y oscuridad (CLO). El aparato constó de dos cámaras de acero inoxidable, una iluminada y una oscura, cada una con dimensiones de 26 × 20 x $19 \mathrm{~cm}$ que se conectaban a través de una abertura de $9 \mathrm{~cm}$ de ancho por $5 \mathrm{~cm}$ de alto, los pisos de ambas cámaras contenían cáscaras de arroz. La luz que incidía sobre la cámara iluminada a través del techo abierto, provenía de tubos fluorescentes de 120 vatios colocados en el techo a 3,5 metros de altura; las paredes y el techo de la cámara oscura estaban cubiertos de cartulina negra. La prueba consistió en colocar al ratón en la cámara iluminada para que explorara libremente ambas cámaras, se cuantificó el número de desplazamientos de una cámara a otra y el tiempo de permanencia en cada cámara, durante un periodo de $10 \mathrm{~min}$.

Prueba de preferencia por sacarosa (PS). Este ensayo se llevó a cabo en jaulas con dimensiones de 30 x 20 x $13 \mathrm{~cm}$. En cada jaula se colocaron dos dispensadores de líquidos de $40 \mathrm{ml}$, uno con $20 \mathrm{ml}$ de agua destilada y otro con $20 \mathrm{ml}$ de solución de sacarosa al 2\%, adicionalmente se colocó en cada jaula 4 grs de comida. Los ratones fueron sometidos a un periodo de adaptación un día previo al ensayo, que consistió en colocarlos individualmente por jaula durante 2 horas con ambos dispensadores y sin comida. Al día siguiente, los ratones fueron colocados nuevamente en las jaulas durante un periodo de 24 horas, midiéndose la cantidad de agua y solución de sacarosa ingerida.

Análisis de los Datos. Los datos son presentados como el promedio de valores absolutos o porcentuales \pm el error o la desviación estándar. Para determinar la significancia estadística de la diferencia observada entre las variables analizadas se utilizó el test de Student no pareado. Los análisis de correlación entre variables cuantitativas fueron realizados mediante el test de Pearson. Se aceptó como significativo una $\mathrm{p}<0.05$. En todos los casos el análisis estadístico fue realizado utilizando el programa GraphPad Prism 6.

Consideraciones Éticas. El proyecto fue aprobado por el Comité de Ética de la Facultad de Ciencias de la Salud, Universidad Centroccidental Lisandro Alvarado, Barquisimeto, Venezuela. La manipulación murina se 
realizó de acuerdo a los criterios éticos para el uso de animales experimentales del Fondo Nacional de Ciencias y Tecnología http:/ /www.fonacit.gov.ve/bioetica.asp\#capitulo1.

\section{RESULTADOS}

Los ratones adaptados a EA (grupo experimental) presentaron un número de entradas significativamente menores a los brazos abiertos y un número de entradas significativamente mayores a los brazos cerrados del LEC en comparación al grupo control (ver Figura 1, panel A y B, Tabla 1). Estos resultados fueron corroborados al observar que, los ratones del grupo experimental se desplazaban con menor frecuencia de un brazo abierto a otro brazo abierto y con mayor frecuencia de un brazo cerrado a otro brazo cerrado (ver Figura 2, panel A y B, Tabla 1). Sin embargo, el tiempo de permanencia total fue similar en los brazos abiertos o en los brazos cerrados, al comparar para cada brazo los ratones de ambos grupos (ver Figura 3, panel A, Tabla 1). La divergencia entre los desplazamientos y el tiempo total se explica, porque los ratones del grupo experimental mostraron hiperactividad locomotora, reflejada en el hecho que presentaron un mayor número de entradas totales a los brazos al ser comparados con los ratones del grupo control (ver Figura 1, panel C, Tabla 1).

En la CLO, los ratones de ambos grupos permanecieron la mayor parte del tiempo (414 segundos; 69\% del tiempo total) en la cámara oscura, validando la ejecución del test (ver Figura 4, panel C, Tabla 1). No obstante, los ratones adaptados a EA (grupo experimental) presentaron un menor número de desplazamientos entre cámaras, al ser comparados con el grupo control (ver Figura 4, panel A, Tabla 1), lo que se tradujo en que los ratones del grupo experimental permanecieron mayor tiempo después de cada desplazamiento, tanto en la cámara oscura como en la iluminada, al ser comparados con el grupo control (ver Figura 4, panel B y C, Tabla 1).

En el test de PS, los ratones con EA mostraron una preferencia significativamente mayor por la sacarosa, al consumir 4,68 \pm 0,71 $\mathrm{ml}$ de la solución al 2\%, en comparación con el grupo control que consumió 1,54 \pm $0,10 \mathrm{ml}$ (ver Figura 4, panel D, Tabla 1).

Finalmente realizamos análisis de correlación entre las diferentes variables cuantificadas para cada individuo en cada grupo experimental por separado. Solo detectamos correlaciones significativas para el grupo GE; encontrando correlaciones positivas entre la motilidad en el LEC y la motilidad en la CLO (ver Figura 5, panel A; Tabla 1), entre el consumo de sacarosa y las entradas a los brazos abiertos en el LEC (ver Figura 5, panel D, Tabla 1) y entre el consumo de sacarosa y el tiempo promedio de permanencia en la cámara oscura de la CLO (ver Figura 5, panel C, Tabla I), mientras que observamos correlación negativa entre el tiempo de permanencia en la cámara oscura de la CLO y el tiempo de permanencia en los brazos cerrados en el LEC (ver Figura 5, panel B, Tabla 1). 
Tabla 1.

Desempeño de ratones C57BL/6 sometidos o no a enriquecimiento ambiental en el laberinto elevado en cruz, cámara luz oscuridad y preferencia por sacarosa

\begin{tabular}{|c|c|c|c|c|}
\hline \multicolumn{2}{|c|}{ Ítems } & \multirow{2}{*}{$\begin{array}{c}\text { Grupos } \\
\rightarrow \text { BA }\end{array}$} & \multirow{2}{*}{$\begin{array}{c}\text { Control } \\
8.10 \pm 0.95\end{array}$} & \multirow{2}{*}{$\begin{array}{c}\text { Experimental } \\
5.44 \pm 0.79 *\end{array}$} \\
\hline \multirow{11}{*}{$\begin{array}{c}\text { Laberinto } \\
\text { Elevado } \\
\text { en Cruz }\end{array}$} & \multirow{7}{*}{ Entradas } & & & \\
\hline & & $\rightarrow \mathrm{BC}$ & $15.20 \pm 2.07$ & $22.30 \pm 1.34 *$ \\
\hline & & Totales & $22.22 \pm 2.47$ & $31.00 \pm 2.67^{*}$ \\
\hline & & $\rightarrow \mathbf{B A}-\mathbf{B A}$ & $7.78 \pm 2.46$ & $0.56 \pm 0.34^{*}$ \\
\hline & & $\rightarrow$ BC-BC & $8.70 \pm 1.92$ & $16.60 \pm 2.04^{*}$ \\
\hline & & $\rightarrow \mathbf{B A}-\mathbf{B C}$ & $7.70 \pm 0.93$ & $5.70 \pm 0.91$ \\
\hline & & $\rightarrow$ BC-BA & $5.30 \pm 0.54$ & $5.70 \pm 0.91$ \\
\hline & Tiempo & $\mathbf{B A}$ & $\begin{array}{c}160.0 \pm \\
40.39\end{array}$ & $73.81 \pm 9.91$ \\
\hline & Total & BC & $\begin{array}{c}440.0 \pm \\
40.39\end{array}$ & $497.7 \pm 29.89$ \\
\hline & Tiempo & $\mathbf{B A}$ & $14.01 \pm 1.42$ & $13.94 \pm 1.15$ \\
\hline & Unitario & $\mathbf{B C}$ & $39.37 \pm 9.69$ & $23.58 \pm 1.13$ \\
\hline \multirow{6}{*}{$\begin{array}{c}\text { Cámara } \\
\text { Luz } \\
\text { Oscuridad }\end{array}$} & \multirow{3}{*}{ Entradas } & CI & $19.30 \pm 1.69$ & $13.90 \pm 1.09 *$ \\
\hline & & $\mathrm{CO}$ & $18.70 \pm 1.75$ & $13.60 \pm 1.10 *$ \\
\hline & & Totales & $38.00 \pm 3.44$ & $25.70 \pm 2.41 *$ \\
\hline & Tiempo & CI & $\begin{array}{c}186.1 \pm \\
32.95\end{array}$ & $185.5 \pm 14.00$ \\
\hline & Total & $\mathrm{CO}$ & $\begin{array}{c}414.0 \pm \\
32.97\end{array}$ & $414.5 \pm 14.01$ \\
\hline & Tiempo & CI & $8.55 \pm 0.98$ & $13.54 \pm 0.79 *$ \\
\hline
\end{tabular}

Nota. $\rightarrow$ : Ingreso; BA: Brazo Abierto; BC: Brazo Cerrado; CI: Cámara Iluminada; CO: Cámara Oscura; (*) Significa $\mathrm{p}<0.05$ al comparar el grupo Experimental con respecto al grupo Control. 
Figura 1. Entradas a los brazos abiertos y brazos cerrados del laberinto elevado en cruz de ratones C57BL/6 sometidos o no a enriquecimiento ambiental
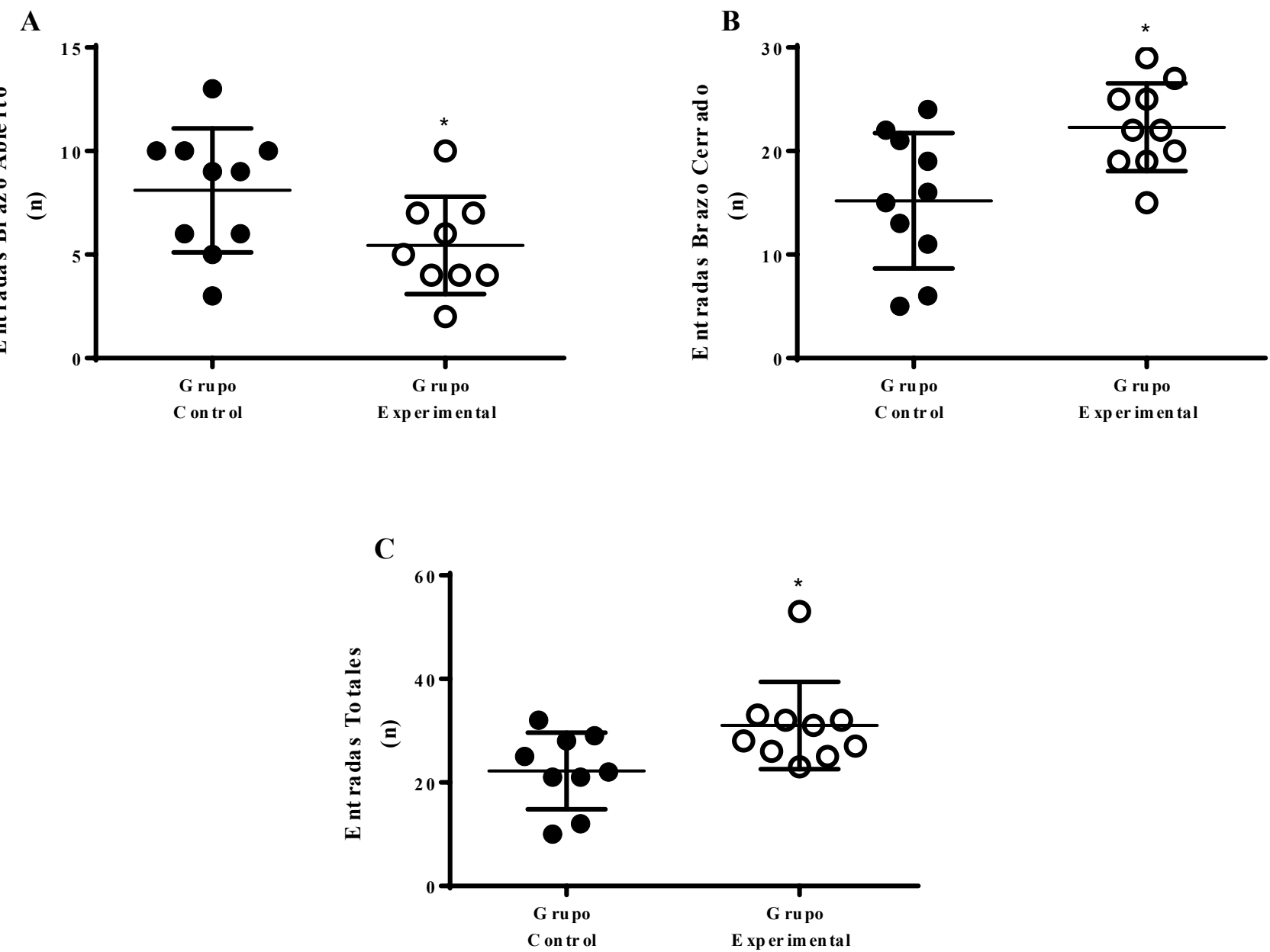

Figura 1. Los ratones fueron colocados individualmente en el brazo abierto para que se desplazaran de manera libre y espontánea durante un periodo de 10 minutos (600 segundos) por todo el laberinto elevado en cruz (LEC), cuantificando las entradas y el tiempo dispensado en cada uno de los brazos. Se observó que los sujetos del grupo experimental (círculos vacíos) presentaron un número de entradas significativamente menor a los brazos abiertos, un número de entradas significativamente mayor a los brazos cerrados (panel A y B) y un número de entradas totales significativamente mayor a los brazos abiertos y cerrados, al ser comparados con los ratones del grupo control (panel C). (*) Significa $(\mathrm{p}<0.05)$ cuando se comparan el grupo experimental con respecto al grupo control. 
Figura 2. Desplazamientos entre los brazos abiertos y entre los brazos cerrados del laberinto elevado en cruz de ratones $\mathrm{C} 57 \mathrm{BL} / 6$ sometidos o no a enriquecimiento ambiental
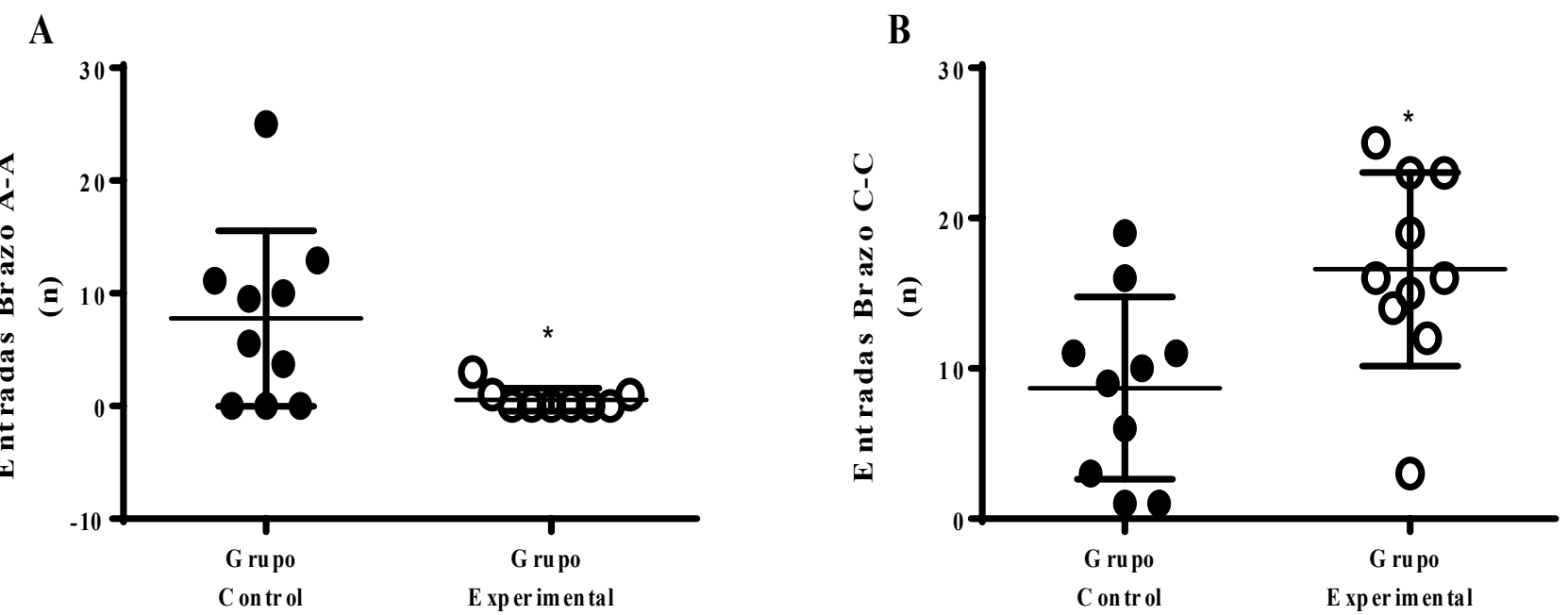

Figura 2. Se cuantificó el número de desplazamientos de un brazo abierto a otro brazo abierto y de un brazo cerrado a otro brazo cerrado de los ratones en el laberinto elevado en cruz (LEC), durante un periodo de 10 minutos (600 segundos). Los ratones del grupo experimental (círculos vacíos) presentaron un número de entradas significativamente menor entre los brazos abiertos (panel A) y un número de entradas significativamente mayor entre los brazos cerrados (panel B), al ser comparado con el grupo control (círculos llenos). (*) Significa $(p<0.05)$ cuando se comparan el grupo experimental con respecto al grupo control.

Figura 3. Tiempo de permanencia en los brazos abiertos y cerrados del laberinto elevado en cruz de los ratones C57BL/6 sometidos o no a enriquecimiento ambiental
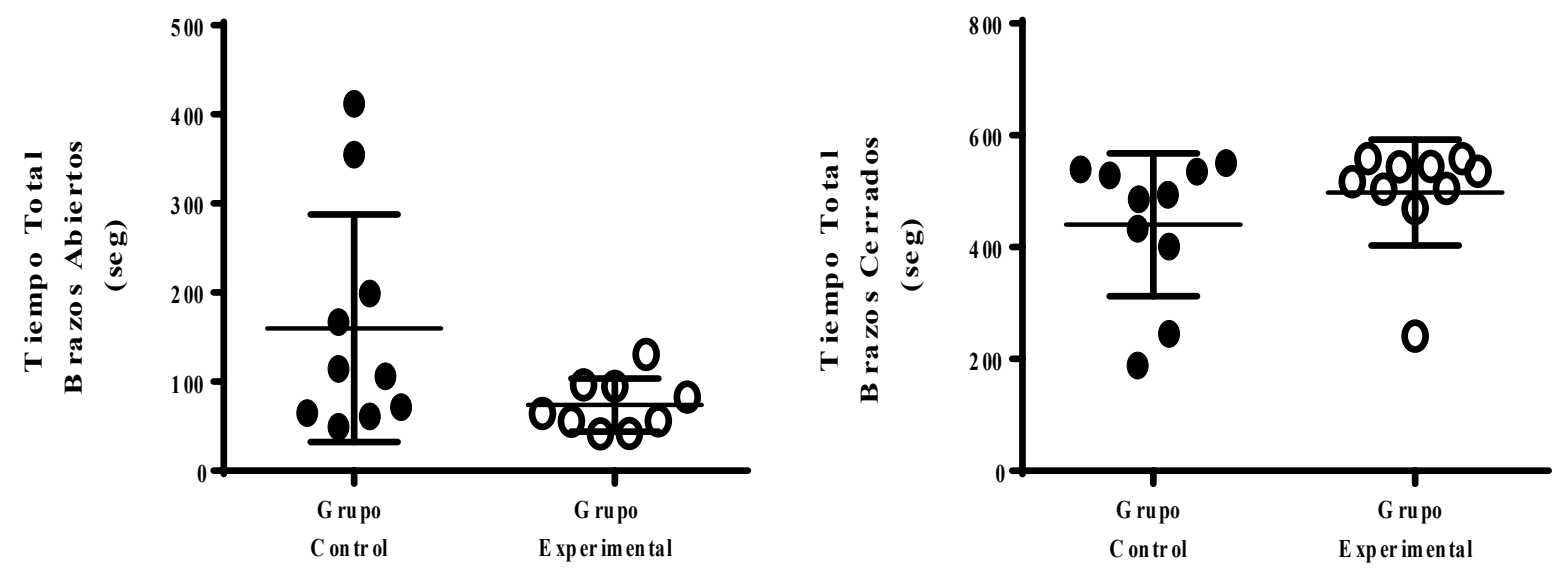

Figura 3. Se tomó el tiempo de permanencia total de cada uno de los sujetos en los brazos abiertos (panel A) y cerrados (panel B) del laberinto elevado en cruz (LEC), durante un periodo de 10 minutos (600 segundos). No se observaron diferencias estadísticamente significativas $(\mathrm{p}>0.05)$ en el tiempo de permanencia total en ninguno de los brazos del laberinto, al comparar el grupo sometido a enriquecimiento ambiental (círculos vacíos) con respecto al grupo control (círculos llenos). 
Figura 4. Desplazamientos y tiempo de permanencia unitario en la cámara luz y oscuridad y preferencia por sacarosa de ratones $\mathrm{C} 57 \mathrm{BL} / 6$ sometidos o no a enriquecimiento ambiental

A

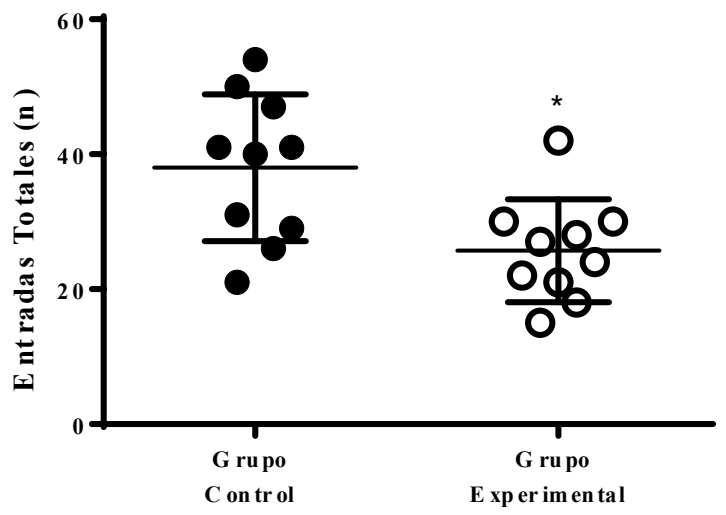

C

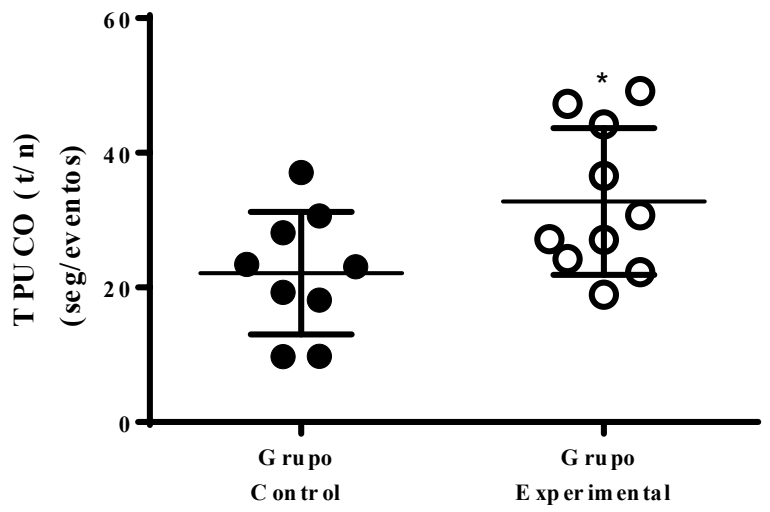

B

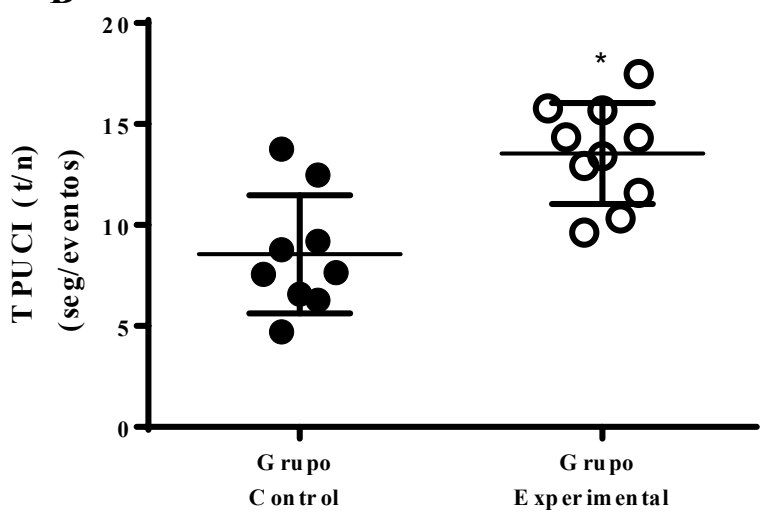

D

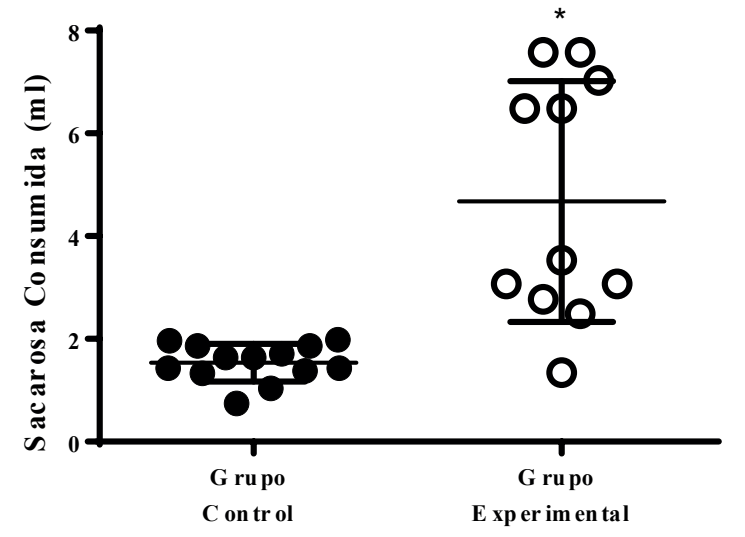

Figura 4. La prueba cámara luz y oscuridad (CLO) consistió en colocar al ratón en la cámara iluminada (CI) para que explorara libremente la cámara oscura (CO) y viceversa, por 10 minutos (600 segundos). Se cuantificó el número de desplazamientos de una cámara a otra y el tiempo de permanencia unitario (TPU) en ellas (tiempo total/número de desplazamientos; $\mathrm{t} / \mathrm{n}$ ). El grupo experimental (círculos vacíos) presento un número de desplazamientos significativamente menor entre cámaras, al ser comparado con el grupo control (círculos llenos), (panel A). Sin embargo, los ratones del grupo experimental permanecieron un tiempo unitario significativamente mayor en ambas cámaras, en comparación con el grupo control (panel B y C). Para la prueba preferencia por sacarosa (PS) el ratón fue colocado individualmente en una jaula que contenía dos dispensadores de líquidos, uno con agua destilada y otro con sacarosa al 2\%, por 24 horas. El grupo experimental consumió un volumen significativamente mayor de sacarosa al ser comparado con el grupo control (Panel D). $\left(^{*}\right)$ Significa $(\mathrm{p}<0.05)$ cuando se comparan el grupo experimental con respecto al grupo control. 
Figura 5. Análisis de correlaciones entre las variables cuantificadas en los ensayos conductuales en ratones $\mathrm{C} 57 \mathrm{BL} / 6$ sometidos o no a enriquecimiento ambiental
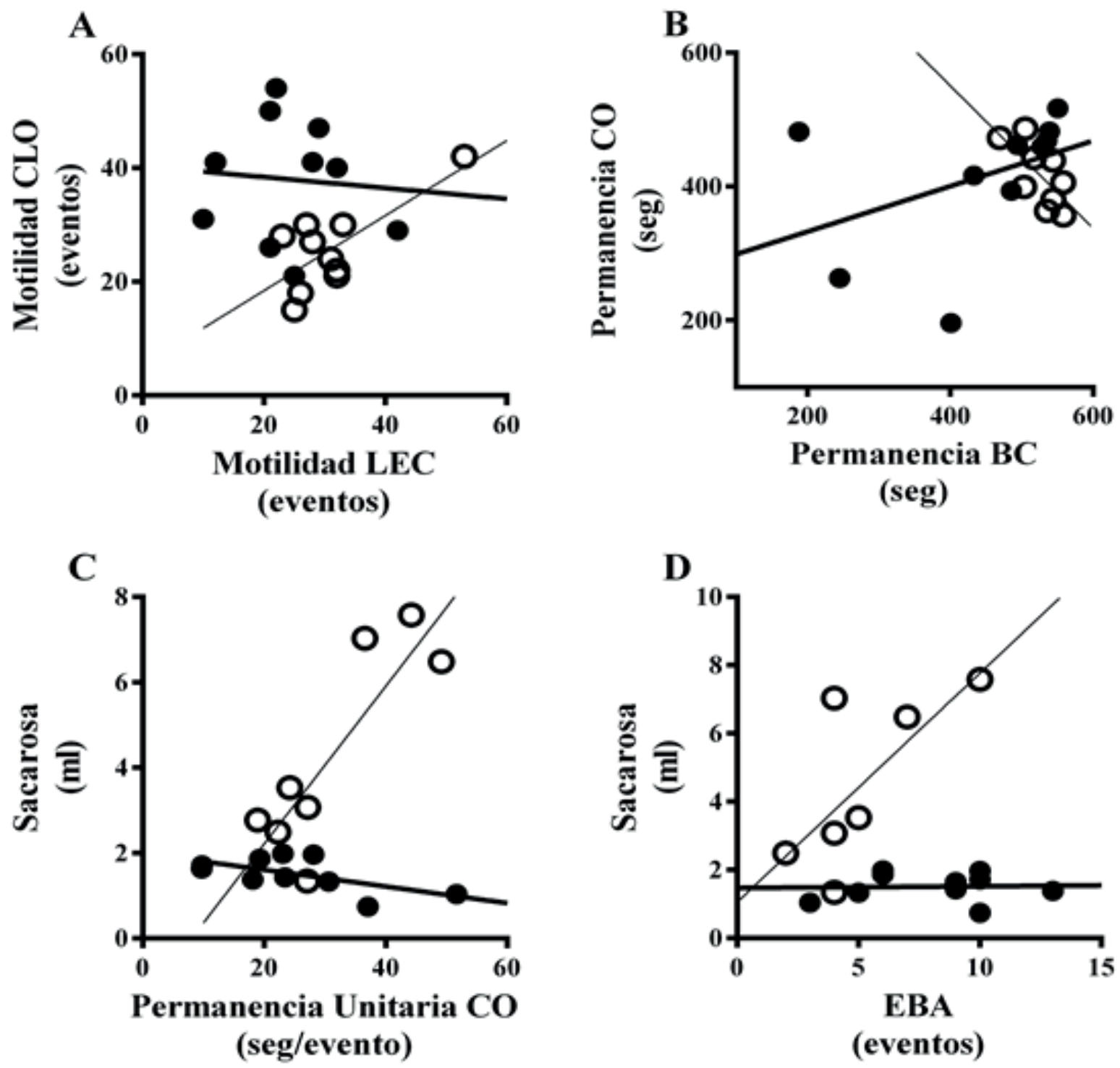

Figura 5. Se observaron correlaciones significativas $(\mathrm{p}<0.05)$ solamente en el grupo sometido a enriquecimiento ambiental (círculos vacíos). Las correlaciones positivas fueron entre el número de entradas totales en ambos compartimientos de la CLO y el número de movimientos entre los brazos abiertos y cerrados del LEC ( $\mathrm{r}$ de Pearson: 0.73; $\mathrm{r}^{2}$ : 0.53; Panel A), entre PS y el tiempo unitario de permanencia en la CO ( $\mathrm{r}$ de Pearson: $0.85 ; \mathrm{r}^{2}$. .72; panel C) y entre PS y las entradas en los brazos abiertos (EBA) del LEC ( $\mathrm{r}$ de Pearson: $0.71 ; \mathrm{r}^{2}$. .5; panel D). Asimismo, se evidenció una correlación negativa entre el tiempo de permanencia en la CO y el tiempo de permanencia en los BC ( $\mathrm{r}$ de Pearson: $-0.68 ; r^{2}: 0.46$; panel B).

\section{DISCUSIÓN}

En el presente trabajo se evidenciaron resultados aparentemente contradictorios, por ejemplo, en el laberinto elevado en cruz observamos que los ratones con EA mostraron una hiperactividad locomotora con preferencia para desplazarse entre los brazos cerrados, mientras que en la CLO su motilidad fue menor entre los brazos y tuvieron mayor preferencia por la sacarosa que por el agua. 
Clásicamente, en el LEC, los animales con altos niveles de ansiedad permanecen mayor tiempo en los brazos cerrados y presentan hipomotilidad, que se traduce en menores desplazamientos entre los brazos cerrados [12]. En el presente trabajo observamos que los ratones con EA tenían una actividad locomotora incrementada con un mayor número de desplazamientos entre brazos cerrados, lo cual inicialmente sugeriría un mayor nivel de ansiedad, sin embargo, la preferencia del animal por desplazarse entre los brazos cerrados o permanecer un mayor tiempo en la cámara oscura, podría ser mejor explicada por la exposición previa de los ratones del grupo enriquecido a un contexto con túneles y madrigueras oscuras, lo que permitió reconocer y relacionar los brazos cerrados como nichos familiares.

Una gran cantidad de roedores están adaptados a ecótopos subterráneos oscuros [13,14]. En concordancia, la existencia de túneles en las jaulas minimiza el estrés en ratones criados en condiciones de laboratorio [15]. En este sentido, Korenman, Watson, \& Silman (1988) [16], mostraron que los ratones alojados en jaulas disimiles interconectadas por túneles, una actuando como una madriguera oscura a prueba de luz y la otra expuesta a la luz, exhibieron una mayor actividad en la madriguera oscura en el ciclo diurno; mientras que por el contrario, una vez que este ciclo finalizó y la intensidad de luz del ambiente disminuyó, los animales comenzaron a pasar períodos de tiempo cada vez mayores en la jaula expuesta. Estos resultados sustentan nuestras observaciones, indicando que el EA con túneles induce hiperactividad y rescata la conducta instintiva de preferencia por desplazarse en lugares oscuros o poco iluminados.

El efecto delEA sobre la actividad locomotora y la ansiedad ha sido previamente explorado, Benaroya-Milshtein et al. (2004) [17], demostraron que ratones criados en condiciones de enriquecimiento ambiental permanecieron más tiempo en los brazos abiertos del laberinto elevado en cruz y subieron escaleras con una mayor frecuencia, resultados que indican una disminución de la ansiedad y una mayor actividad motora. De la misma manera, Rogers, Li, Lanfumey, Hannan y Renoir (2017) [18], ensayando en el LEC y en la CLO, ratones con deleción del gen para el transportador de serotonina (modelos animales para la ansiedad y depresión), encontraron que el EA mejoró la ansiedad innata en ambas pruebas, sin mejorar el comportamiento similar a la depresión en el nado forzado.

La no preferencia por desplazarse entre brazos abierto-abierto observadas en el presente trabajo, no sería indicativo de una respuesta ansiosa sino de una respuesta a la cual el animal no estaba previamente entrenado, esto se corrobora por el hecho de que las entradas a los brazos abiertos en los ratones con EA se correlacionaron positivamente con el consumo de sacarosa, indicando que los animales no presentaban ansiedad para entrar en los brazos abiertos, sino que estaban motivados por una conducta anti-depresiva.

La prueba de PS es una prueba basada en recompensas, utilizada como indicador de la disminución de la capacidad de experimentar placer (anhedonia), la cual representa uno de los síntomas centrales de la depresión. Los roedores nacen interesados en los alimentos o soluciones dulces, la preferencia reducida por la solución dulce representa la anhedonia, esta reducción puede revertirse mediante el tratamiento con antidepresivos [19]. En el presente trabajo, el EA indujo preferencia por el consumo de sacarosa que se correlacionó positivamente con las entradas a los brazos abiertos, confirmando el efecto ansiolítico y antidepresivo del EA. Similarmente, Brenes, Villagra y Fornaguera (2006) [20], concluyeron que el EA ejercía efectos antidepresivos y ansiolíticos evaluados mediante la prueba de nado forzado, la preferencia por sacarosa y el test de campo abierto.

Por el contrario, en la CLO, los ratones con EA mostraron menos actividad locomotora y permanecieron más tiempo en ambas cámaras, con preferencia en la cámara oscura; esto último se correlacionó positivamente con un mayor consumo de sacarosa, indicando que los animales con EA sentían mayor placer de permanecer 
en la cámara oscura, en concordancia a los espacios a los cuales estaban acostumbrados y en concordancia con sus conductas instintivas [13-16].

La aparente contradicción entre las motilidades observadas en el LEC y la CLO, es un problema de probabilidades por las opciones presentadas. En el primero siempre existió la opción de desplazarse al brazo cerrado contralateral, mientras que en el segundo esa opción no existió, más aún en nuestro protocolo experimental no se midió la motilidad dentro de la cámara oscura. El hecho de que el tiempo de permanencia en la cámara oscura se correlacionará negativamente con el tiempo de permanencia en los brazos cerrados, indica que la conducta en los brazos cerrados refleja hipermotilidad con niveles ansiedad disminuidos, lo cual en concordancia se refleja en el menor tiempo de permanencia en la cámara oscura.

Los resultados obtenidos en el presente trabajo, pueden ser productos del protocolo de EA utilizado. Diversos autores han encontrado que animales sometidos a ambientes enriquecidos tuvieron un mayor nivel de actividad locomotora, comparados con los animales hospedados en ambientes estándar, efecto que estuvo relacionado con la fase del día [21-22]. Por el contrario, otros autores reportaron que ratones o ratas alojadas en condiciones de EA no mostraron diferencias o fueron menos activas en el campo abierto en relación a los animales controles [23]. Las diferencias observadas han sido relacionadas con las características físicas (tamaño, forma y color) del equipo utilizado, al protocolo ensayado (simples o repetitivos), el ambiente donde fue ejecutado el ensayo (intensidad de la luz y niveles de ruido), la duración del ensayo, la cepa y sexo de los animales utilizados [21-22-24]. Sin embargo, el EA tiende a mejorar más drásticamente la actividad locomotora en ratones viejos [25] o en modelos animales de enfermedades con déficit neurológico [26].

Por otra parte, Christakis, Benedikt, Ferguson, Ravindera y Ramirez (2018) [27], desarrollaron un modelo animal de excesiva estimulación sensorial, mediante el uso de distractores auditivos y luces de colores. Ellos demostraron que la ansiedad, el aprendizaje y la memoria disminuyeron en estos ratones, mientras que la toma de riesgos y los niveles de actividad locomotora aumentaron. En el presente trabajo, además de enriquecer el ambiente, incluimos estimulación táctil, olfativa, gustativa y visual, variable o intermitente, por lo que planteamos que nuestro esquema experimental podría ser un modelo de EA con rasgos concretos de sobre-estimulación sensorial. Este punto de vista, permitiría explicar la hiperactividad y el déficit de ansiedad observados.

\section{CONCLUSIÓN}

En el presente trabajo se demostró que el EA basado en incluir túneles oscuros en su protocolo, induce hiperactividad con niveles de ansiedad y depresión disminuidos, lo cual permite que las conductas instintivas de preferencia por lugares poco iluminados sean expresadas; esto implica que la interpretación de los resultados obtenidos en el laberinto elevado en cruz y en la caja luz/oscuridad son dependientes del tipo de hospedaje o entrenamiento previos del animal.

\section{FINANCIAMIENTOS}

Los autores declaramos que no recibimos ningún tipo de apoyo financiero de ninguna institución pública ni privada.

\section{CONFLICTO DE INTERESES}

Los autores declaramos que no existen ningún tipo de conflictos de intereses relacionados con la generación, utilización y divulgación de los datos obtenidos, autorizando la publicación del mismo. 


\section{AGRADECIMIENTOS}

Suárez-Rodríguez MA y Pulido-Barrera SP pertenecen al programa de Talento Científico Juvenil de la Unidad de Bioquímica, Consejo de Desarrollo Científico y Humanístico y Tecnológico (C.D.C.H.T), Universidad Centro Occidental Lisando Alvarado.

\section{REFERENCIAS}

1. Organización Mundial de la Salud. Desórdenes mentales, 2018. Disponible en: https://www.who.int/news-room/fact-sheets/detail/mental-disorders

2. Organización Panamericana de la Salud. La carga de los trastornos mentales en la Región de las Américas, 2018. Washington, D.C.: OPS; 2018.

3. Steimer T. Animal models of anxiety disorders in rats and mice: some conceptual issues. Dialogues Clin Neurosci. 2011;13(4):495-506. https://doi.org/10.31887/DCNS.2011.13.4/tsteimer. PMid:22275854 PMCid:PMC3263396

4. American Psychiatric Association - APA. Manual diagnóstico y estadístico de los trastornos mentales DSM-5 (5a. ed.). 2014. Madrid: Editorial Médica Panamericana.

5. Norman TR, Ellen SR, Burrows GD. Benzodiazepines in anxiety disorders: managing therapeutics and dependence. Med J Aust. 1997 Nov 3;167(9):490-5. https://doi.org/10.5694/j.1326-5377.1997.tb126684.x . PMid:9397065

6. Bandelow B, Michaelis S. Epidemiology of anxiety disorders in the 21st century. Dialogues Clin Neurosci. 2015 Sep 17(3):327-35. https://doi.org/10.31887/DCNS.2015.17.3/bbandelow PMid:26487813 PMCid:PMC4610617

7. Sampedro-Piquero P, Begega A. Environmental Enrichment as a Positive Behavioral Intervention Across the Lifespan. Curr Neuropharmacol. 2017;15(4):459-470. https://doi.org/10.2174/1570159X14666160325115909. PMid:27012955 PMCid:PMC5543669

8. Slater AM, Cao L. A Protocol for Housing Mice in an Enriched Environment. J Vis Exp. 2015 Jun 8;(100):e52874. doi: 10.3791/52874. PMID: 26131694; PMCID: PMC4544994.

9. Suárez Rodríguez MA, Pulido Barrera SP, Caicedo Pinto PJ, Bonfante Cabarcas RA. Efecto del enriquecimiento ambiental sobre la actividad locomotora, habituación, aprendizaje y memoria en ratones C57BL/6. Gac. Cs. Vet. [Internet]. 15 de julio de 2019 [citado 21 de enero de 2022];24(1):8-20. Disponible en: https://revistas.uclave.org/index.php/gcv/article/view/3208

10. Lopes D, Cespedes I, Viana M. Effects of environmental enrichment on anxiety measurements: A review. Research and Reviews: Neuroscience. 2017;2(1):1-6.

11. Ball NJ, Mercado E III, Orduña I. Enriched environments as a potential treatment for developmental disorders: a critical assessment. Psychol 2019; 10:466. https:/ / doi.org/10.3389/fpsyg.2019.00466 PMid:30894830 PMCid:PMC6414413

12. Pellow S, Chopin P, File SE, Briley M. Validation of open:closed arm entries in an elevated plus-maze as a measure of anxiety in the rat. J Neurosci Methods. 1985 Aug;14(3):149-67. PMID: 2864480. https://doi.org/10.1016/0165-0270(85)90031-7

13. Faulkes, C. Mosaic evolution of subterranean mammals - Regression, progression and global convergence. Heredity. 2000 Apr 84: 493-494. https://doi.org/10.1046/j.1365-2540.2000.0726b.x

14. Lacey E, Patton JL, Cameron G. Life Underground: The Biology Of Subterranean Rodents. Australian Mammalogy. 2001. Jan 23(1): 75-76. https://doi.org/10.1071/AM01075_BR

15. Gouveia K, Hurst JL. Reducing mouse anxiety during handling: effect of experience with handling tunnels. PLoS One. 2013 Jun 20;8(6):e66401. https://doi.org/10.1371/journal.pone.0066401. PMid:23840458 PMCid:PMC3688777

16. Korenman EM, Watson BW, Silman RE. Activity rhythms of hamsters in a single cage compared to a simulated burrow system. Physiol Behav. 1988;43(4):459-69. PMID: 3194466.

https://doi.org/10.1016/0031-9384(88)90120-5 
17. Benaroya-Milshtein N, Hollander N, Apter A, Kukulansky T, Raz N, Wilf A, et al. Environmental enrichment in mice decreases anxiety, attenuates stress responses and enhances natural killer cell activity. Eur J Neurosci. 2004 Sep;20(5):1341-7. https://doi.org/10.1111/j.1460-9568.2004.03587.x . PMid:15341605

18. Rogers J, Li S, Lanfumey L, Hannan AJ, Renoir T. Environmental enrichment reduces innate anxiety with no effect on depression-like behaviour in mice lacking the serotonin transporter. Behav Brain Res. 2017 Aug 14;332:355-361. doi: 10.1016/j.bbr.2017.06.009. Epub 2017 Jun 16. PMID: 28629963. https://doi.org/10.1016/j.bbr.2017.06.009. PMid:28629963

19. Serchov T, van Calker D, Biber K. Sucrose preference test to measure Anhedonic Behaviour in Mice. Bio-protocol. 2016; 6(19). https:// doi.org/10.21769/BioProtoc.1958

20. Brenes Sáenz JC, Villagra OR, Fornaguera Trías J. Factor analysis of Forced Swimming test, Sucrose Preference test and Open Field test on enriched, social and isolated reared rats. Behav Brain Res. 2006 Apr 25;169(1):57-65. https:/ /doi.org/10.1016/j.bbr.2005.12.001. PMid:16414129

21. Loss CM, Binder LB, Muccini E, Martins WC, de Oliveira PA, Vandresen-Filho S, Prediger RD, Tasca CI, Zimmer ER, Costa-Schmidt LE, de Oliveira DL, Viola GG. Influence of environmental enrichment vs. time-of-day on behavioral repertoire of male albino Swiss mice. Neurobiol Learn Mem. 2015 Nov;125:63-72. https:// doi.org/10.1016/j.nlm.2015.07.016. PMid:26247375

22. Sudo M, Nagamatsu T, Ando S. Does environmental enrichment increase locomotor activity in rats? Evidence from an implanted sensor device., Bulletin of the Physical Fitness Research Institute. 2018; 116, 29-32.

23. Brenes JC, Padilla M, Fornaguera J. A detailed analysis of open-field habituation and behavioral and neurochemical antidepressant-like effects in postweaning enriched rats. Behav Brain Res. 2009 Jan 30;197(1):125-37. https://doi.org/10.1016/j.bbr.2008.08.014 PMid:18786573

24. Toth LA, Kregel K, Leon L, Musch TI. Environmental enrichment of laboratory rodents: the answer depends on the question. Comp Med. 2011 Aug;61(4):314-21. PMID: 22330246; PMCID: PMC3155397.

25. McMurphy T, Huang W, Queen NJ, Ali S, Widstrom KJ, Liu X, Xiao R, Siu JJ, Cao L. Implementation of environmental enrichment after middle age promotes healthy aging. Aging (Albany NY). 2018 Jul 20;10(7):1698-1721. https://doi.org/10.18632/aging.101502. PMid:30036185 PMCid:PMC6075449

26. Jankowsky JL, Melnikova T, Fadale DJ, Xu GM, Slunt HH, Gonzales V, Younkin LH, Younkin SG, Borchelt DR, Savonenko AV. Environmental enrichment mitigates cognitive deficits in a mouse model of Alzheimer's disease. J Neurosci. 2005 May 25;25(21):5217-24.

https://doi.org/10.1523/JNEUROSCI.5080-04.2005. PMid:15917461 PMCid:PMC4440804

27. Christakis D, Julian S. Benedikt Ramirez, S. Ferguson, S. Ravinder, Jan-Marino Ramirez et al. "How early media exposure may affect cognitive function: A review of results from observations in humans and experiments in mice." Proceedings of the National Academy of Sciences 115 (2018): 9851 - 9858. https://doi.org/10.1073/pnas.1711548115 . PMid:30275319 PMCid:PMC6176595 